International Joumal of Biological Sciences
2009; 5(2):128-134

(C) Ivyspring International Publisher. All rights reserved

Research Paper

\title{
Assay Validation for KIM-l: human urinary renal dysfunction biomarker
}

\author{
Shalini Chaturvedi, Takeisha Farmer, and Gordon F. Kapke ${ }^{\bowtie}$ \\ Department of Biomarker Services, Covance Central Laboratory Services Inc., 8211 SciCor Drive, Indianapolis, Indiana \\ 46214-2985, USA
}

$\triangle$ Correspondence to: Gordon F. Kapke, Ph.D., Tel.: 317-273-7901; Fax: 317-273-7990; E-mail: gordon.kapke@covance.com

Received: 2008.10.17; Accepted: 2009.01.17; Published: 2009.01.19

\begin{abstract}
Urinary kidney injury molecule (KIM-I) is a sensitive quantitative biomarker for early detection of kidney tubular injury. The objective of the present work was to analytically validate this urinary renal injury biomarker. Duo-set reagents from R\&D were used to develop the ELISA and validate the assay's linearity, intra-run precision, inter-run precision, lower limit of quantification, recovery, dilutional verification, reference range, stability, and length of run. The reference range data suggests that the healthy population falls within the assay range (59 - $2146 \mathrm{pg} / \mathrm{mL}$ ) and upper limit of quantitation for this assay is $17168 \mathrm{pg} / \mathrm{mL}$ for the patient population. This is a robust assay to detect urinary levels of $\mathrm{KIM}-\mathrm{I}$, which serves as a non-invasive sensitive, reproducible, and potentially high-throughput method to detect early kidney injury in drug development studies.
\end{abstract}

Key words: KIM-1; ELISA; urinary biomarker; validation.

\section{Introduction}

Acute kidney injury (AKI) has been defined as a rapid decline in glomerular filtration rate that occurs over hours or days $[1,2]$. AKI is common, and absolute incidence of AKI has increased [3], diagnosis of which is frequently based on measurements of blood urea nitrogen (BUN) [4]. BUN and serum creatinine are not very specific or sensitive for the diagnosis of AKI because they are affected by many renal and non-renal (age, sex, race, muscle mass, nutritional status, infection, and volume of distribution, certain medications) [5-7] factors that are independent of kidney injury or kidney function.

Clearly, there is a need to identify a more specific AKI biomarker that is produced at the site of injury, can be measured easily in blood or urine and should be reasonably stable in body fluids to substantially improve the diagnosis. Several biomarkers have been studied in animal models and human of AKI [4], and kidney injury molecule-1 (KIM-1) is a recently discovered tubular protein that is markedly induced after ischemic reperfusion injury and in re- sponse of a number of other proximal tubular toxins, such as cisplatin and folic acid [8,9]. KIM-1 is a type I transmembrane glycoprotein, with an ectodomain containing Ig-like domain and a mucin domain [8]. It is undetectable in healthy kidney tissue, but expressed at very high levels in dedifferentiated proximal tubule epithelial cells in human and rodent kidneys after ischemic or toxic injury $[8,10]$.

Hence, KIM-1, the new biomarker of kidney injury and glomerular filtration, holds the promise of substantially improving the diagnostic approach to acute kidney injury $[9,11]$. Previously, an ELISA has been designed and validated for quantitating KIM-1 in rat urine as an animal model, but this first generation ELISA has not been tested on human urine [12]. Subsequently, an ELISA has been developed previously to measure KIM-1 in human urine [13]. These KIM-1 reagents are not commercially available, therefore there is a need to develop, evaluate, and validate a high-throughput commercially available method for quantifying KIM-1 in human urine. Ana- 
lytically validating KIM-1 would provide clinical studies with the means to quantify the KIM-1 biomarker so that it can be evaluated for clinical practice. Therefore, the goal of the study was to analytically validate KIM-1 in human urine and demonstrate appropriate clinical performance using the ELISA from a R\&D duo-set [14].

This assay is a Sandwich ELISA based on: 1) uniformly coating goat anti-human KIM-1 in the micro-plate well, followed by washing and then blocking plates non-specifically, 2) binding of human KIM-1 in the sample to the immobilized KIM-1 antibody, 3) the binding of a biotinylated goat anti-human KIM-1 detection antibody, 4) wash away of unbound materials, followed by conjugation of streptavidin-horseradish peroxidase to the immobilized biotinylated antibodies, 5) wash away of free enzyme, and 6) quantification of immobilized antibody-enzyme conjugates by monitoring horseradish peroxidase activities in the presence of the substrate $3,3^{\prime}, 5,5^{\prime}$-tetra-methylbenzidine. The enzyme activity is measured spectrophotometrically by increased absorbance at $450 \mathrm{~nm}$, corrected from the absorbance at $540 \mathrm{~nm}$, after acidification of formed products. Since the increase in absorbance is directly proportional to the amount of captured KIM-1 in the unknown sample, the concentration of KIM-1 can be derived by interpolation from a reference curve generated in the same assay with reference standards of known concentrations of human KIM-1 [14]. The standard curve concentrations used for all the KIM-1 ELISAs were $2000,1000,500,250,125,62.5$, and $31.25 \mathrm{pg} / \mathrm{mL}$ and the absorbances read approximately $2.5,1.5,0.9,0.5$, $0.25,0.12$, and 0.05 respectively. The curve fit for the analysis of the standard curve was four parametric logistic (4PL).

\section{Materials and Methods}

Sample collection from Healthy population: For the determination of the reference interval, blood and urine samples were collected from healthy healthcare professionals (10 males and 10 females). Individuals were informed of the testing to be performed and only the specified testing was performed on the collected reference specimens. Analyses were conducted with the full respect for the individual's right to confidentiality. Information regarding their age, gender and race was also collected with the healthcare professional's consent to calculate the glomerular filtration rate (GFR). Urine creatinine and serum creatinine was assayed for each of these samples. Freshly collected urine samples were allowed to sit at room temperature for 30 minutes to sediment, and the supernatant was aliquoted and stored at $-70^{\circ} \mathrm{C}$ until analysis.
Construction of KIM-1 sandwich ELISA (RED Cat\# DY1750, Minneapolis, MN): The wells of Nunc-Maxisorp EIA plates were coated by diluting the capture antibody $(72 \mu \mathrm{g} / \mathrm{mL})$ to a working concentration of $0.4 \mu \mathrm{g} / \mathrm{mL}$ in PBS with $100 \mu \mathrm{L}$ in each well. The plate was sealed and incubated overnight at room temperature. Each well was aspirated and washed using an automated microplate washer (Bio-Tek) with $400 \mu \mathrm{L}$ of Wash Buffer $(0.05 \%$ Tween-20 in PBS), repeating the process two times for a total of three washes. The plates were blocked by adding $300 \mu \mathrm{L}$ of reagent diluent (1\% BSA in PBS, 0.2 $\mu \mathrm{m}$ filtered) to each well and incubated at room temperature for 2 hours. After washing as in the previous step, $100 \mu \mathrm{L}$ of standard recombinant human KIM-1 $(0-2000 \mathrm{pg} / \mathrm{mL})$, control, and urine sample was pipetted to the designated well, covered with an adhesive strip, and placed on the orbital shaker at $400 \mathrm{rpm}$ at room temperature for 2 hours. The plate was washed using the same wash protocol as before, and $100 \mu \mathrm{L}$ of the biotinylated goat anti-human KIM-1 detection antibody diluted in reagent diluent to a working concentration of $400 \mathrm{ng} / \mathrm{mL}$ was added to each well. The plate was covered with a new adhesive strip, incubated at room temperature for 2 hours with continued shaking at $400 \mathrm{rpm}$. Washing step was repeated and $100 \mu \mathrm{L}$ of streptavidin-HRP diluted to a working dilution was added to each well. The plate was protected from light, covered with a new adhesive strip, shaken at $400 \mathrm{rpm}$, and incubated at room temperature for 20 minutes. After washing, $100 \mu \mathrm{L}$ substrate solution was added to all wells, protected from light, covered with an adhesive strip, shaken at $400 \mathrm{rpm}$, and incubated at room temperature for 7 minutes. The reaction was stopped by adding $50 \mu \mathrm{L}$ of stop solution to all wells. The absorbance was measured using a plate reader (BioTek Elx800) at $450 \mathrm{~nm}$ with an absorbance correction at $540 \mathrm{~nm}$. The urinary KIM-1 concentration was calculated based on the standard curve and expressed in absolute terms (pg/mL).

Evaluation of the KIM-1 ELISA: The validation of the KIM-1 ELISA was evaluated by measuring linearity, intra-run precision, inter-run precision, analytical sensitivity, recovery, dilution verification, reference range, stability and length of run. Details of the criteria for each are described in the result section.

Sample collection and analysis: Urine samples were collected asceptically directly in urine cups and stored at $-70{ }^{\circ} \mathrm{C}$ within 4 hours. All statistical analysis was carried out using the program EP evaluator [15] (version 8.0, DG Rhoads).

Analytical Testing: Urinary creatinine was measured by the Jaffe rate-blanked creatinine assay using a 
Roche/ Hitachi 911 system (Roche Diagnostics, Indianapolis, IN, USA). Serum enzymatic creatinine was measured using the Roche/Hitachi P800 analyzer. Glomerular filteration rate (GFR) was calculated using the MDRD (modification of diet in renal disease) formula $186 \mathrm{X}$ (Serum Creatinine $^{-1.154} \mathrm{X}(\text { Age })^{-0.203} \mathrm{X}(0.742$ if female and 1.000 if male) $X(1.210$ if African American and 1.000 if others) $\left(\mathrm{mL} / \mathrm{min} / 1.73 \mathrm{~m}^{2}\right)$ [16].

\section{Results}

Linearity: In order to know the range of the assay, it is important to define the linear range of the assay. To define the range, a high standard was serially diluted with reagent diluent to give a series of specimens $(100+0,75+25,50+50,25+75$, and $0+100)$. Each sample was assayed for KIM-1 in duplicates (N $=4)$, and slope calculated. The plotted linearity shows a slope of 1.051, and the data demonstrates that the assay is acceptably linear to $2146 \mathrm{pg} / \mathrm{mL}$ (Figure 1).

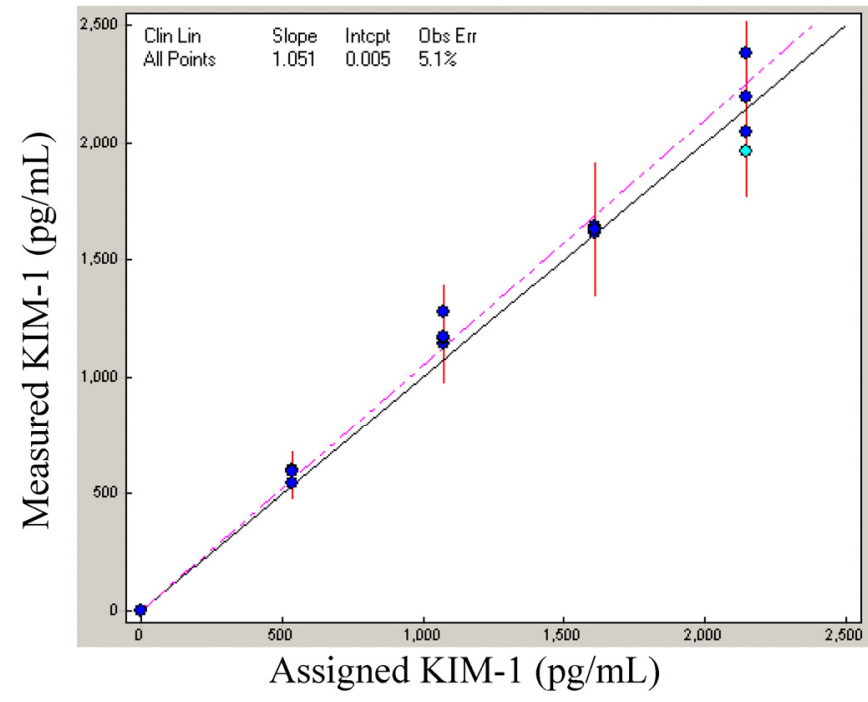

Figure I: Linearity: KIM-I linearity was demonstrated with the high standard diluted proportionately with buffer.

Precision: Two sets (low and high) of pooled urine samples were created and each set was run on two separate plates for $\mathrm{N}=20, \% \mathrm{CV}$ calculated for each to give intra-low and intra-high precision. The intra-low sample gave an average reading of $170 \pm 11$ $\mathrm{pg} / \mathrm{mL}(\mathrm{CV}=6.4 \%, \mathrm{~N}=20)$, while the intra-high sample averaged $2007 \pm 98 \mathrm{pg} / \mathrm{mL}(\mathrm{CV}=4.9 \%, \mathrm{~N}=$ 20). Low and high pools were also run on 8 separate plates, and \% CV calculated to give an inter-low and inter-high precision (Table 1). The mean low value for inter-low precision was $164 \pm 10 \mathrm{pg} / \mathrm{mL}(\mathrm{CV}=6.0 \%$, $\mathrm{N}=8$ ), and that of inter-high precision was $383 \pm 15$ $\mathrm{pg} / \mathrm{mL}(\mathrm{CV}=3.8 \%, \mathrm{~N}=8)$.
Table I: KIM-I assay precision

\begin{tabular}{|c|c|c|c|c|}
\hline Sample & $\mathrm{N}$ & Mean $(\mathrm{pg} / \mathrm{mL})$ & $\mathrm{SD}$ & $\mathrm{CV}(\%)$ \\
\hline Intra Low & 20 & 170 & 11 & 6.4 \\
\hline Intra High & 20 & 2007 & 98 & 4.9 \\
\hline Inter Low & 8 & 164 & 10 & 6.0 \\
\hline Inter High & 8 & 383 & 15 & 3.8 \\
\hline
\end{tabular}

Sensitivity: The assay's lower limit of quantification (LLOQ) is defined as the lowest concentration at which an across run \% CV can be generated that is less than $20 \%$. A series of samples in the concentration range of 57 to $384 \mathrm{pg} / \mathrm{mL}$ were generated to provide multiple low samples that were aliquoted and frozen. Samples were thawed and analyzed on 10 separate plates over several days. The $\% \mathrm{CV}$ of each sample was determined from the 10 analytical runs and the lowest sample that demonstrated inter-assay precision (\%CV) of less than $20 \%$ was the LLOQ of the assay. The LLOQ for the assay as read from the curve is defined as $59 \mathrm{pg} / \mathrm{mL}(\% \mathrm{CV}=19.8)$.

Recovery: In order to establish the \% recovery, 5 urine samples were identified that read between 120 $550 \mathrm{pg} / \mathrm{mL}$ and one high sample that read 1805 $\mathrm{pg} / \mathrm{mL}$. A second set of 5 samples were generated by mixing $10 \%$ of the high sample with $90 \%$ of the low samples. These samples were then assayed for KIM-1 activity. Based on the observed concentration of KIM-1 for the original 5 samples, the expected values were calculated. The average recovery of the samples was found to be $89.4 \%$ (Table 2).

Table 2: KIM-I recovery; where I:I 0 represents a mixture of $90 \%$ of sample and $10 \%$ of spike. The expected value was compared with the observed value and recovery calculated.

\begin{tabular}{|c|c|c|c|}
\hline Sample & Observed & Expected & $\%$ Recovery \\
\hline Spike & 1805 & & \\
\hline 1 & 120 & & \\
\hline 2 & 134 & & \\
\hline 3 & 420 & & \\
\hline 4 & 417 & & \\
\hline 5 & 550 & & \\
\hline $10 \%$ spike $+90 \% 1$ & 299 & 289 & 103.7 \\
\hline $10 \%$ spike $+90 \% 2$ & 275 & 302 & 91.4 \\
\hline $10 \%$ spike $+90 \% 3$ & 411 & 559 & 73.5 \\
\hline $10 \%$ spike $+90 \% 4$ & 510 & 556 & 91.8 \\
\hline $10 \%$ spike $+90 \% 5$ & 584 & 676 & 86.5 \\
\hline
\end{tabular}


Dilution Verification: The dynamic range for the measurement of KIM-1 has been defined as $59-2146$ $\mathrm{pg} / \mathrm{mL}$. This range poses difficulty for samples that are higher than the defined range. The problem is typically resolved by diluting the sample so that the reading falls within the defined range. The reading is then multiplied with the dilution factor to generate the true value. This is valid only if the dilution process is linear. A high sample $(694.40 \mathrm{pg} / \mathrm{mL})$ was diluted $1: 2,1: 4,1: 8,1: 16,1: 32$, and 1:64 in reagent diluent and each diluted reading was assayed for \% recovery. The high sample diluted appropriately to $1: 8$, with $87.0 \%$ recovery, demonstrating that the high sample can be diluted at least 8 fold, indicating the upper limit of quantification (upper limit of quantification = upper limit of linearity $X$ highest dilution allowed) to be $17168 \mathrm{pg} / \mathrm{mL}$ (2146 X 8) (Figure 2).

Reference Range: Urine and serum was collected from 20 healthy donors (10 males and 10 females), assayed for urinary KIM-1, urinary creatinine and serum creatinine. KIM-1 ranged from $60-837$ pg/mL, and when normalized to urinary creatinine, the results spanned the range of $0.070-0.399$

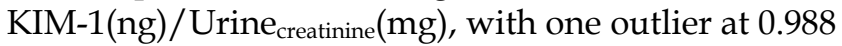

(Figure 3). The MDRD calculated GFR for all the volunteers was $>65.00$ (Table 3 ).

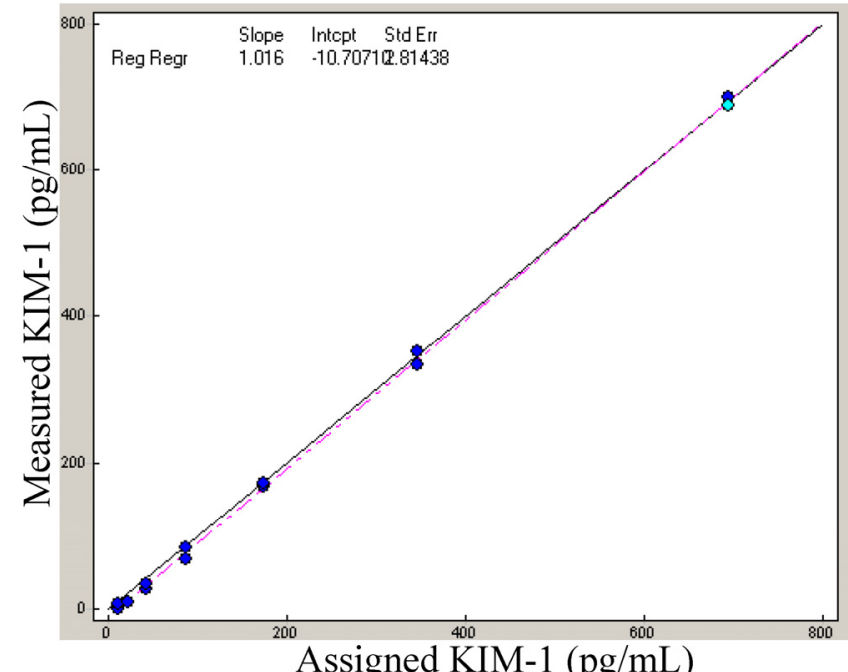

Figure 2: Dilution Verification: A urine specimen with a high endogenous KIM-I concentration was serially diluted with reagent diluent, assayed and \% recovery calculated. Most diluted samples recovered between $80-120 \%$ of expected concentration.

Table 3: Summary of baseline characteristics of healthy population.

\begin{tabular}{|c|c|c|c|c|c|c|c|c|}
\hline Sample & $\begin{array}{c}\text { Serum } \text { Creatinine } \\
(\mathbf{m g} / \mathbf{d L})\end{array}$ & $\begin{array}{c}\text { Age } \\
\text { (Years) }\end{array}$ & $\begin{array}{c}\text { African } \\
\text { American? }\end{array}$ & Gender & $\begin{array}{c}\text { GFR } \\
\left(\mathrm{mL} / \mathrm{min} / 1.73 \mathrm{~m}^{2}\right)\end{array}$ & $\begin{array}{l}\text { Urine }_{\text {Creatinine }} \\
(\mathbf{m g} / \mathrm{dL})\end{array}$ & $\begin{array}{c}\text { KIM-1 } \\
(\mathrm{pg} / \mathrm{mL})\end{array}$ & $\begin{array}{c}\text { KIM-1/Urine } \\
\text { (ng/mg) }\end{array}$ \\
\hline S01 & 0.79 & 29 & $\mathrm{~N}$ & F & 91 & 164.3 & 355 & 0.216 \\
\hline $\mathrm{S} 02$ & 0.71 & 39 & Y & $\mathrm{F}$ & 118 & 142.8 & 396 & 0.277 \\
\hline $\mathrm{S} 03$ & 0.74 & 28 & Y & $\mathrm{F}$ & 120 & 156.0 & 273 & 0.175 \\
\hline S04 & 0.69 & 42 & $\mathrm{~N}$ & $\mathrm{~F}$ & 99 & 197.9 & 561 & 0.283 \\
\hline S05 & 0.83 & 32 & Y & $\mathrm{F}$ & 103 & 96.2 & 339 & 0.353 \\
\hline S06 & 1.02 & 59 & Y & F & 71 & 234.8 & 336 & 0.143 \\
\hline S07 & 0.71 & 33 & $\mathrm{~N}$ & $\mathrm{~F}$ & 101 & 147.6 & 165 & 0.112 \\
\hline S08 & 0.62 & 31 & $\mathrm{~N}$ & $\mathrm{~F}$ & 119 & 84.7 & 837 & 0.988 \\
\hline S09 & 0.69 & 46 & $\mathrm{~N}$ & $\mathrm{~F}$ & 97 & 30.0 & 120 & 0.399 \\
\hline S10 & 0.79 & 42 & $\mathrm{~N}$ & $\mathrm{~F}$ & 85 & 121.3 & 379 & 0.312 \\
\hline S11 & 1.05 & 46 & $\mathrm{~N}$ & $\mathrm{M}$ & 81 & 11.1 & 27 & 0.243 \\
\hline S12 & 0.78 & 34 & $\mathrm{~N}$ & M & 121 & 156.7 & 248 & 0.158 \\
\hline $\mathrm{S} 13$ & 1.01 & 24 & $\mathrm{~N}$ & M & 96 & 132.1 & 524 & 0.396 \\
\hline S14 & 0.99 & 30 & $\mathrm{~N}$ & M & 94 & 57.4 & 60 & 0.104 \\
\hline S15 & 1.09 & 28 & $\mathrm{~N}$ & M & 86 & 166.6 & 333 & 0.200 \\
\hline S16 & 1.03 & 30 & Y & $\mathrm{M}$ & 109 & 151.7 & 107 & 0.070 \\
\hline S17 & 1.05 & 31 & $\mathrm{~N}$ & M & 88 & 158.9 & 305 & 0.1 .92 \\
\hline S18 & 0.98 & 22 & $\mathrm{~N}$ & M & 102 & 198.0 & 440 & 0.222 \\
\hline S19 & 1.14 & 26 & $\mathrm{~N}$ & M & 83 & 42.0 & 83 & 0.198 \\
\hline $\mathrm{S} 20$ & 1.07 & 40 & $\mathrm{~N}$ & M & 81 & 221.8 & 605 & 0.273 \\
\hline
\end{tabular}

*GFR was calculated using the formula

GFR $\left(\mathrm{mL} / \mathrm{min} / 1.73 \mathrm{~m}^{2}\right)=186 \mathrm{X}\left(\text { Serum }_{\text {Creatinine }}\right)^{-1.154} \mathrm{X}(\text { Age })^{-0.203} \mathrm{X}(0.742$ if female and 1.000 if male $) \mathrm{X}(1.210$ if African American and 1.000 if others) 


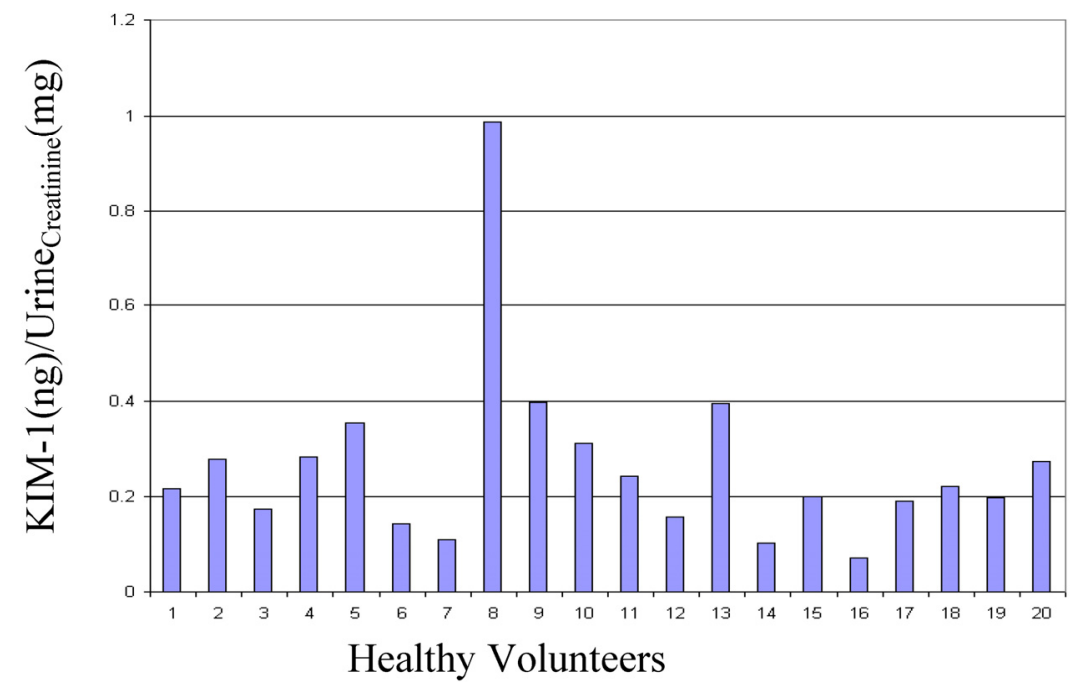

Figure 3: Reference Interval: Urine specimens collected from 20 healthy subjects was assayed to determine the reference range of KIM-I. Values for healthy volunteers ranged from 60 - $837 \mathrm{Pg} / \mathrm{mL}$, and the ratios $\mathrm{KIM}-\mathrm{I}(\mathrm{ng} / \mathrm{mL}) /$ Urine $_{\text {Creatinine }}(\mathrm{mg} / \mathrm{mL})$ ranged from $0.070-0.399$, with one outlier at 0.988 .

Length of run: In order to make sure that there was no plate effect, and the samples read the same on any well in the plate, we placed QC samples at the end of lanes 6, 9 and 12. The SD calculated during the intra-run precision was $11 \mathrm{pg} / \mathrm{mL}(\mathrm{CV}=6.4 \%)$ for intra-low, and $98 \mathrm{pg} / \mathrm{mL}(\mathrm{CV}=4.9 \%)$ for intra-high. The $3 \mathrm{SD}$ range was established and applied to the QC samples on the plate. The range was calculated to be $142-210 \mathrm{pg} / \mathrm{mL}$ for the low and $350-471 \mathrm{pg} / \mathrm{mL}$ for the high QC samples. QC samples in lanes 6, 9, and 12 fell within range confirming the length of run experiment.

Stability: To test the stability of the samples over time, freeze-thaw cycles, and storage temperatures (room temperature, $\left.4,-20,-70^{\circ} \mathrm{C}\right)$, aliquoted samples $(\mathrm{N}=6)$ in 21 different tubes were subjected to a host of conditions. The \% recovery was compared to the baseline value for all 6 samples. KIM-1 was found to be stable up to 7 days refrigerated and ambient conditions. Samples recovered within 99-114 \% when subjected to 3 freeze-thaw cycles, and also at $-20^{\circ} \mathrm{C}$, and $-70^{\circ} \mathrm{C}$ at 14 days (Figure 4). Stability studies up to 1 year are under way.

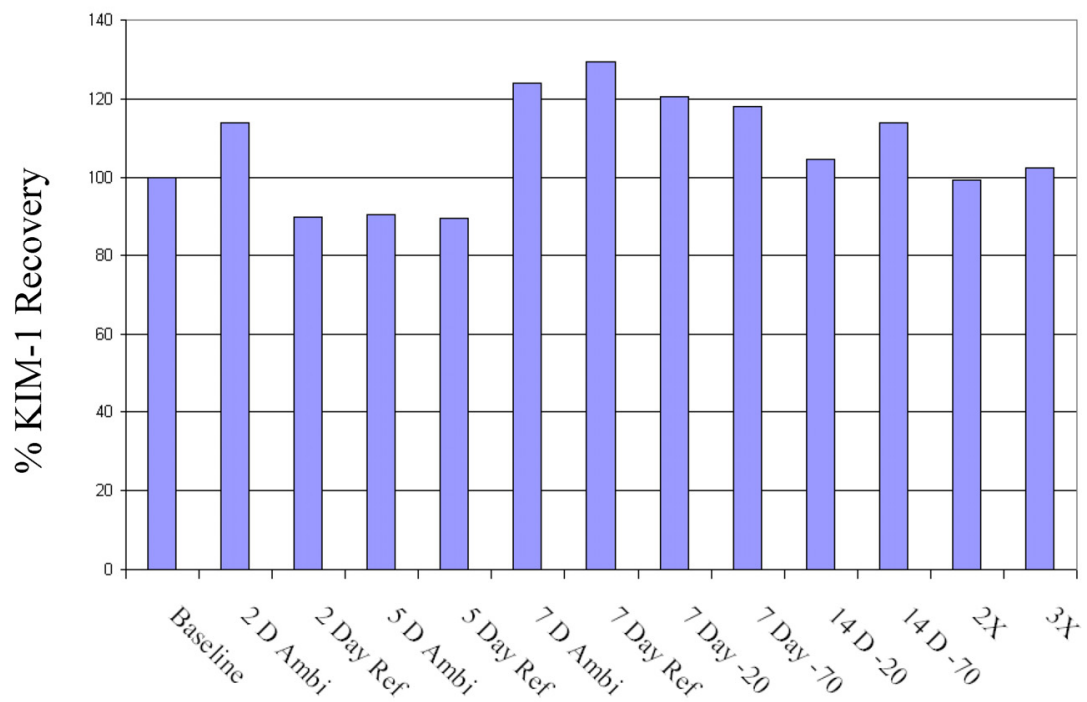

Figure 4: Stability: Recovery of KIM-I at ambient, refrigerated, -20 , and $-70^{\circ} \mathrm{C}$ over 14 days; and 2 and 3 times freeze-thaw was compared to the baseline samples $(\mathrm{N}=6)$. The plotted data is the mean value for the 6 samples. The mean value for sample I- 6 across different conditions is $56.44 \pm 33.22$, $253.43 \pm 37.43,668.62 \pm 199.19,315.29 \pm 35.15,396.76 \pm 77.6 \mathrm{I}$, $401.66 \pm 51.63 \mathrm{pg} / \mathrm{mL}$. Sample I reading 56.44 is below the LLOQ, therefore a high standard deviation is not unusual. 


\section{Discussion}

The US FDA and European Medicines Agency (EMEA) have completed a major milestone under their Critical Path and Roadmap to 2010 through an extensive process of data generation and analysis and "qualified" seven new nephrotoxicity biomarkers for use in regulatory decision-making. This consortium of public-private partnership led by the Critical Path Institute, generated and submitted the data for seven urinary proteins- KIM-1, albumin, total protein, $\beta 2$-microglobulin, cystatin $C$, clusterin, and trefoil factor-3. Efforts are underway to qualify these markers for human studies and clinical trials $[17,18]$. The evaluated product is the only commercially available KIM-1 assay to support broad use of this newly recognized regulatory biomarker.

There is a growing body of evidence that supports KIM-1 as a specific marker for ischemic renal injury, and therefore a sensitive, validated assay to measure urinary levels of KIM-1 is required for clinical research. A rigorous validation of the KIM-1 assay to optimize the performance for high-throughput routine clinical research was performed. The commercially available KIM-1 assay described in this paper is suitable to assay KIM-1 in human urine.

This paper reports development of a commercially available quantitative assay for measuring KIM-1 in human urine. This assay could have direct implications for evaluation of nephrotoxicity in humans where reliance on serum creatinine is questioned. A significant advantage of this KIM-1 assay is the ease of use, analytical sensitivity, and rapid throughput, making the assay reliable for diagnostic and prognostic measurements. All the solutions are prepared easily using commercially available buffers and the recombinant KIM-1 protein was used as the assay standard. The KIM-1 assay has dynamic range of $0-2146 \mathrm{pg} / \mathrm{mL}$ and a lower limit of quantification of $59.0 \mathrm{pg} / \mathrm{mL}$ with inter-assay variability of $19.8 \%$. The performance characteristics of the ELISA are consistent over time, making it a robust assay for research and clinical research use. This study, for the first time, gives us data on the short-term stability of the KIM-1. The long-term stability data is being generated as well. The experiment on dilution verification show appropriate recovery up to a 1:8 dilution, indicating no interference in the urine matrix from any other factors. This is further confirmed by the spike recovery results giving approximately $90 \%$ recovery.

The only other human KIM-1 data has been published by Han et. al [10]. Their normalized KIM-1 levels in normal healthy individuals were less than those of other acute renal failure patients at $0.48 \pm 0.19$

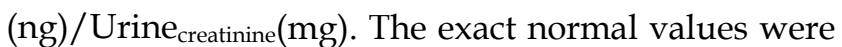
not published. The determined reference range (mean $\pm 2 \mathrm{SD}$ ) in our lab with 19 donors (one outlier removed) was $0.228 \pm 0.188$ (ng)/Urine creatinine $(\mathrm{mg})$, and including the outlier was $0.266 \pm 0.386$

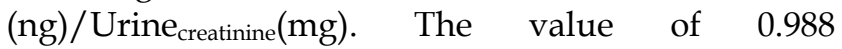
(ng)/Urine creatinine $(\mathrm{mg})$ was well above the 3SD range, which is why it was considered an outlier.

Investigators in the past have relied on multiple classical laboratory tests to detect AKI, but the biggest disadvantage these tests have is time delay between injury and detection making them marginally effective biomarkers. Some of the disadvantages current biomarkers face are: lack of stability, lack of stability due to $\mathrm{pH}$ of urine which means the analyte has to be neutralized soon after collection, need to add stabilizers soon after collection, inhibition due to metals, lack of specificity, and onset of elevation and sustainability. KIM-1 has excellent frozen stability and the literature indicates excellent sensitivity to AKI. The KIM-1 ELISA assay also provides the throughput to handle large number of samples over extended period of time.

In conclusion, analytical validation of the KIM-1 assay demonstrates this assay can be used reliably and routinely in a standard clinical research setting. The analytical performance of this assay permits meaningful comparison of data between trials. KIM-1 has a great potential as a biomarker of renal injury, and the R\&D duo set assay provides the ability for any researcher to generate KIM-1 data.

\section{Acknowledgement}

We would like to thank the staff of Covance Central Laboratories, Indianapolis for providing the healthy urine, and serum specimens in this study to verify the reference range.

\section{Conflict of Interest}

The authors have declared that no conflict of interest exists.

\section{References}

1. Lassnigg A, Schmidlin D, Mouhieddine M, Bachmann LM, Druml W, Bauer P, Hiesmayr M. Minimal changes of serum creatinine predict prognosis in patients after cardiothoracic surgery: a prospective cohort study. J Am Soc Nephrol. 2004; 15(6): 1597-605.

2. VanBiesen $W$, Vanholder R, Lameire N. Defining acute renal failure: RIFLE and beyond. Clin J Am Soc Nephrol. 2006; 1(6): 1314-19.

3. Waikar SS, Curhan GC, Wald R, McCarthy EP, Chertow GM. Declining mortality in patients with acute renal failure, 1988 to 2002. J Am Soc Nephrol. 2006; 17(4): 1143-50.

4. Edelstein CL. Biomarkers of acute kidney injury. Adv Chronic Kidney Dis. 2008; 15(3): 222-34. 
5. Stevens LA, Lafayette RA, Perrone RD, et al. Laboratory evaluation of kidney function. In: Schrier RW, ed. Diseases of the Kidney and Urinary Tract $8^{\text {th }}$ ed. Philadelphia, PA: Lippincott, Williams and Wilkins; 2007.

6. Star RA. Treatment of acute renal failure. Kidney Int. 1998; 54(6): 1817-31.

7. Vaidya VS, Ferguson MA, Bonventre JV. Biomarkers of acute kidney injury. Annu Rev Pharmacol Toxicol. 2008; 48: 463-93.

8. Ichimura T, Bonventre JV, Bailly V, Wei H, Hession CA, Cate RL, Sanicola M. Kidney injury molecule-1 (KIM-1), a putative epithelial cell adhesion molecule containing a novel immunoglobulin domain, is up-regulated in renal cells after injury. J Biol Chem. 1998; 273(7): 4135-42.

9. Ichimura T, Hung CC, Yang SA, Stevens JL, Bonventre JV. Kidney injury molecule-1: a tissue and urinary biomarker for nephrotoxicant-induced renal injury. Am J Physiol Renal Physiol. 2004; 286(3): F552-63.

10. Han WK, Bailly V, Abichandani R, Thadhani R, Bonventre JV. Kidney Injury Molecule-1 (KIM-1): a novel biomarker for human renal proximal tubule injury. Kidney Int. 2002; 62(1): 237-44.

11. Bonventre JV. Kidney Injury Molecule-1 (KIM-1): a specific and sensitive biomarker of kidney injury. Scand J Clin Lab Invest Suppl. 2008; 241: 78-83.

12. Vaidya VS, Ramirez V, Ichimura T, Bobadilla NA, Bonventre JV. Urinary kidney injury molecule-1: a sensitive quantitative biomarker for early detection of kidney tubular injury. Am J Physiol Renal Physiol. 2006; 290(2): F517-29.

13. Vaidya VS, Ramirez V, Ichimura T, Bobadilla NA, Bonventre JV. Urinary kidney injury molecule-1: a sensitive quantitative biomarker for early detection of kidney tubular injury. Am J Physiol Renal Physiol. 2006; 290(2): F517-29.

14. R\&D Systems Inc. Minneapolis, MN, USA. KIM-1 assay kit instructions for use (ed 751761; 5/08). R\&D Systems Inc: Minneapolis, MN, USA. 2008

15. [Internet] Rhoads DG. http://www.dgrhoads.com

16. [Internet] NKDEP. http://www.nkdep.nih.gov/professionals/ gfr_calculators/orig_con.htm

17. [Internet] CPATH. http://www.c-path.org/pdf/PSTC_nephro _VXDS_summary_final.pdf

18. [Internet] FDA. http://www.fda.gov/bbs/topics/NEWS/2008 /NEW01850.html 